\title{
Characterization of Particles by Means of Local Observables
}

\author{
Volker Enss \\ Abteilung für Theoretische Physik, Fakultät für Physik der Universität Bielefeld, D-4800 Bielefeld 1, \\ Federal Republic of Germany
}

\begin{abstract}
We introduce the notion of singly localized states and use it to characterize the one-particle states as those states which are singly localized at all times. For theories which satisfy the Haag-Swieca compactness criterion, we show that a state has a discrete mass spectrum if and only if it is a "geometrical one-particle state".

Using a mathematical description of coincidence arrangements of counters we show that in asymptotically complete theories the asymptotic particle number is the asymptotic number of localization centres.
\end{abstract}

\section{Introduction}

In relativistic quantum theory a particle is usually defined to be a state that belongs to an irreducible representation of the Poincaré group. In an experiment, however, one identifies a particle by its localization properties, e.g. its track in a bubble chamber. A geometrical characterization of a one-particle state should allow for the experimental situation.

Apart from being very abstract, the usual particle definition has other drawbacks: In theories with long-range forces, like quantum electrodynamics, it is an open question whether the electron has a discrete mass or whether it is an "infraparticle" with continuous mass distribution [6]. In the latter case it would violate the usual particle definition, although it behaves like a particle in experiments.

In theories with short-range forces, the Haag-Ruelle scattering theory [5] provides the existence of states which can be interpreted as incoming or outgoing particle configurations. It relies on the conventional notion of a particle. It is not yet clear which physically plausible assumptions ensure an asymptotic particle interpretation of all states (asymptotic completeness).

As a contribution to these problems, we will characterize the particle states by their local properties, because the physically and mathematically basic objects in the theory are the local observables. We confine ourselves to theories with short-range forces.

In what follows, a "particle" will be any physical system which remains connected for all times when external forces are absent, i.e. a system that does not decay into subsystems which become separated and independent of each other. A particle of this kind can be a stable elementary particle, or a stable bounded 
system like the ground state of a hydrogen atom. Although the possibly existing different components of the particle remain close to each other, the centre of mass motion of the particle exhibits the quantum phenomenon of "spreading": the region where the particle can be found increases with time.

Therefore the notion of a state that is "localized in some region at time $t$ " cannot be used to distinguish the one-particle states from the others. For our purposes, the appropriate generalization is the notion of a state which is "singly localized at time $t$ with correlation-radius $r$ ". These states can be constructed by superposition of state-vectors which are localized at time $t$ in various regions of radius $r$. Alternatively, the singly localized states can be characterized by their inability to trigger a coincidence-arrangement of two counters separated by a longer distance than $r$. In non-relativistic quantum mechanics, a system of $n$ elementary particles is singly localized with correlation radius $r$ at a given time if its wave function $f\left(t ; \boldsymbol{x}_{1}, \ldots, \boldsymbol{x}_{n}\right)$ vanishes as soon as one of the relative coordinates becomes bigger than $2 r$.

Accordingly, an $N$-fold localized state will trigger a coincidence arrangement of $N$ separated counters, but not one with $N+1$ counters.

Loosely speaking, a one-particle state is singly localized for all times. The precise statement of this is the

\section{Geometrical Particle Definition:}

$\Psi$ is a one-particle state if for any $\varepsilon>0$ there is a radius $r$, independent of $t$, such that $\left\|\Psi-\exp (i H t) \Phi_{t}\right\|<\varepsilon$, where $\exp (i H t) \Phi_{t}$ are suitable states singly localized at time $t$ with correlation radius $r$.

Similarly, we define the

\section{Geometrical Asymptotic Particle Number:}

A state is an outgoing (incoming) $N$-particle state if it is $N$-fold localized for $t \rightarrow \infty(-\infty)$.

In Section $\mathrm{V}$ we will check the equivalence of the geometrical particle definition with the usual one. The "compactness criterion" postulated by Haag and Swieca [3] plays an essential role. It says roughly that there are only a finite number of distinct states which are localized in some region and are bounded in energy. In a theory which satisfies this criterion, both notions of a particle are equivalent. The typical behaviour of a one-particle state in space and time entails its discrete mass and vice versa. The proof of the equivalence theorem depends very little on the details of the construction of singly localized states. Therefore a generalization to theories with massless particles might be possible.

The mathematical properties of "coincidence operators", and the equivalence of the asymptotic particle number with the asymptotic number of localization centres will be discussed in Sections VI-IX for asymptotically complete quantum field theories.

The framework for our investigations is the relativistic local quantum theory according to Haag-Araki [1]. An open region of Minkowski space is denoted by $\mathcal{O}$, and $\mathscr{R}(\mathcal{O})$ is the von Neumann algebra generated by the observables of this region. $U(x)=\exp \left\{i \stackrel{\sim}{P}^{\mu} x_{\mu}\right\}$ is a unitary, strongly continuous representation of the 
translations on the Hilbert space $\mathscr{H}$. The spectrum of their generators is contained in the set

$$
\left\{p^{\mu}=0\right\} \cup\left\{p^{\mu} \mid p^{0}>0, p^{\mu} p_{\mu} \geqq \kappa^{2}>0\right\} .
$$

The vacuum $\Omega$ is unique.

\section{IIa. Localized States}

Haag and Swieca described states which are at some time localized in a fixed region of space. We will state their definition and some relevant properties; further details can be found in [3].

We confine our attention to the vacuum sector of the theory. The generalization to theories with superselection rules is obvious if one uses the local fieldalgebras instead of the observable-algebras.

Let $\mathcal{O}$ be a fixed bounded region in space-time. We use local operators $Q$ with the following properties:

$$
Q \in \mathscr{R}(\mathcal{O}),(\Omega, Q \Omega)=0,\|Q\| \leqq \exp (\kappa r)\|Q \Omega\|,
$$

( $\kappa$ is the lower bound of the mass spectrum) to create from the vacuum the set $\mathscr{M}_{r}$ of states which are at time $t=0$ localized in a region of radius $r$ around the origin:

$$
\mathscr{M}_{r}=\{Q \Omega \mid Q \text { satisfies }(2.1)\} \text {. }
$$

$\mathscr{M}_{r}$ is not a linear subspace of the Hilbert space, but

$$
\Psi \in \mathscr{M}_{\boldsymbol{r}} \Rightarrow \lambda \Psi \in \mathscr{M}_{\boldsymbol{r}} ; \quad \mathscr{M}_{\boldsymbol{r}} \subset \mathscr{M}_{\boldsymbol{r}^{\prime}} \quad \text { if } r<r^{\prime} ;
$$

and given two vectors $\Phi, \Psi \in \mathscr{M}_{r}$ there is an $r^{\prime}$ such that

$$
\begin{aligned}
& \lambda \Phi+\mu \Psi \in \mathscr{M}_{r^{\prime}} \text { for all } \lambda, \mu . \\
& \Omega \oplus \bigcup_{r} \mathscr{M}_{r}=\{\mathscr{R}(\mathcal{O}) \Omega\} \text { is dense in } \mathscr{H}
\end{aligned}
$$

by the Reeh-Schlieder theorem (e.g. [1], Part I, Satz (10.2)).

The states which are localized at time 0 in $n$ widely separated regions are

$\prod_{i=1}^{n} Q_{i}\left(\boldsymbol{x}_{i}\right) \Omega, \quad\left|\boldsymbol{x}_{i}-\boldsymbol{x}_{j}\right| \gg r$,

where all $Q_{i}$ satisfy (2.1).

The localization of the states shows up in the following properties:

1. There are numbers $A$ and $\alpha>0$ such that for all $\Phi, \Psi \in \mathscr{M}_{r}$

$$
|(\Phi, U(\boldsymbol{x}) \Psi)| \leqq\|\Phi\|\|\Psi\| A \exp \{-\alpha(|\boldsymbol{x}|-r)\}
$$

2. Any state in $\mathscr{M}_{r}$ is almost orthogonal to any state which is localized in several regions.

3. Let $C$ be a "counter-operator" [cf. (6.10)], i.e. an almost local operator which annihilates the vacuum; then for any $r$ there is a rapidly decreasing function $\varphi_{r}(x)$ such that

$$
|(\Psi, C(\boldsymbol{x}) \Psi)| \leqq\|\Psi\|^{2} \varphi_{r}(|x|) \forall \Psi \in \mathscr{M}_{r} .
$$


To prove (2.6) one uses the local approximations of $C(\boldsymbol{x})$ that lie spacelike to the creator of $\Psi$.

The only strictly localized states are those which are created from the vacuum by local isometric operators. All other states differ from the vacuum even at spacelike distances from $\mathcal{O}$, but the amplitude of this difference falls off very fast with increasing distance. So it is impossible to fix a unique region of localization for the states in $\mathscr{M}_{r} ; r$ is not an exact radius, but a non-calibrated parameter for the spacelike extension of the state.

\section{IIb. The Compactness Criterion}

Haag and Swieca set up a compactness criterion in order to characterize asymptotically complete theories by their local properties [3].

A closed bounded subset $\mathfrak{f}$ of a Hilbert space is compact (in the strong topology) if it is almost finite dimensional, i.e. for any $\varepsilon>0$ there is a finite dimensional projector $F$ with

$$
\|\Psi-F \Psi\|<\varepsilon \forall \Psi \in \mathfrak{l} .
$$

The states of a physical system which are localized in some finite region and have bounded energy occupy a finite volume $\Gamma$ of phase space. Quantum physics says that there is only a finite number of these states, namely $\Gamma / h^{3}$ : Amrein and Georgescu [8] showed in non-relativistic scattering theory that the localized states with finite energy form a compact set (for all realistic potentials). Taking this over to relativistic quantum theory yields the compactness criterion:

$$
\mathfrak{f}_{r, E}=\left\{P_{E} \Psi \mid \Psi \in \mathscr{M}_{r},\|\Psi\| \leqq 1\right\}^{-} \quad \text { is compact } \forall r, E .
$$

$\mathscr{M}_{r}$ contains the localized states with radius $r(2.2), P_{E}$ is the projection on the states with energy smaller than $E$, and \{\}$^{-}$denotes the closure of \{\} . We won't need the postulates on the size of the compact sets given in [3].

The free massive field satisfies this criterion, which means that for a system of free particles there is essentially a finite number of states with bounded energy which are localized in a finite region. The generalized free field, however, which obeys all the axioms except asymptotic completeness [7], violates the criterion.

In an interacting asymptotically complete theory with short-range forces, the localized states of finite energy evolve into states of freely moving particles. To a given accuracy, the interaction between the particles can be neglected after some finite time interval. Since the states are then localized in some bigger but still finite region, there should not be more than a finite number of these states. Therefore one expects that an asymptotically complete theory satisfies the compactness criterion, although this conjecture has not yet been proved. As we are interested in theories with particle interpretation, we shall assume in the sequel that $(2.8)$ is fulfilled.

\section{Separation of the Momentum Distribution}

We will decompose the state space into a direct integral of eigenspaces of the momentum operator $\underset{\sim}{\boldsymbol{P}}$ (in quantum mechanics this is the same as separation of the centre of mass coordinate). This representation is useful for describing the 
translation invariant sets of singly localized states, and it facilitates use of the time evolution to distinguish between states with discrete and continuous mass.

The vectors $\Phi$ with $\int d^{3} x|(\Phi, U(\boldsymbol{x}) \Phi)|<\infty$ are dense in the orthogonal complement of the vacuum. For any given $p \in \mathbb{R}^{3}$, the pairs $(\Phi, \boldsymbol{p})$ with $\lambda(\Phi, p)+$ $\mu\left(\Phi^{\prime}, \boldsymbol{p}\right)=\left(\lambda \Phi+\mu \Phi^{\prime}, \boldsymbol{p}\right)$ span a linear space which can be equipped with the positive semidefinite scalar product

$$
\left\langle(\Phi, \boldsymbol{p}),\left(\Phi^{\prime}, \boldsymbol{p}\right)\right\rangle=\int d^{3} x \exp (i \boldsymbol{p} \boldsymbol{x})\left(\Phi, U(\boldsymbol{x}) \Phi^{\prime}\right) .
$$

A standard mathematical procedure (as described e.g. in [9, Chapter 3.4]) yields a Hilbert space $h_{\boldsymbol{p}}$ for any $\boldsymbol{p} . \Phi(\boldsymbol{p}) \in h_{\boldsymbol{p}}$ denotes the equivalence class of pairs $(\Phi, p)$.

The momentum operator acts on $h_{\mathbf{p}}$ according to

$$
(U(\boldsymbol{x}) \Phi)(\boldsymbol{p})=\exp (-i \boldsymbol{p} \boldsymbol{x}) \Phi(\boldsymbol{p}) .
$$

The Hilbert space is decomposed into ${ }^{1}$

$$
\begin{aligned}
& \mathscr{H}=\{\lambda \Omega\} \oplus \int_{\mathbb{R}^{3}} \oplus d^{3} p h_{\boldsymbol{p}}, \\
& \|\Psi\|_{\mathscr{H}}^{2}=|(\Psi, \Omega)|^{2}+\int d^{3} p\|\Psi(\boldsymbol{p})\|_{h}^{2} .
\end{aligned}
$$

For an $L^{2}$-function there is a unique continuous representative $\tilde{f}(\boldsymbol{p})$ if its Fouriertransform $f(\boldsymbol{x})$ is in $L^{1}$. So the mapping $\tilde{f} \rightarrow \tilde{f}(\boldsymbol{p})$ makes sense. Similarly the above construction gives a unique mapping $\mathscr{H} \rightarrow h_{p}: \Phi \rightarrow \Phi(p)$ for any $p$ and all $\Phi$ with $\int d^{3} x|(\Phi, U(\boldsymbol{x}) \Phi)|<\infty$, although two functions $\Phi(\boldsymbol{p})$ and $\Psi(\boldsymbol{p})$ represent the same vector in $\mathscr{H}$ if they coincide for almost all $p$.

Using this unique mapping it is easy to show that the strongly continuous unitary group of time translations induces on each $h_{p}$ a strongly continuous unitary group. It defines the positive self-adjoint "reduced" Hamiltonian and mass operator with

$$
H[p]=\left(p^{2} \mathbb{1}+M^{2}[p]\right)^{1 / 2} .
$$

To characterize the singly localized states, we will need the following lemma.

Lemma 3.1. In a theory which satisfies the compactness criterion (2.8), the sets

$$
\left\{\left(P_{M} \Psi\right)(q) \mid \Psi \in \mathscr{M}_{r},\|\Psi\| \leqq c\right\}^{-}
$$

are compact in $h_{\boldsymbol{q}}$ for all $M, \mathbf{q}, r$, and $c$.

$P_{M}$ is the projection onto states with mass bounded by $M$.

Proof. For any $\Psi \in \mathscr{M}_{r},\|\Psi\| \leqq c$, there is a $Q \in \mathscr{R}(\mathcal{O}),\|Q\| \leqq c l^{\kappa r}$ with $\Psi=Q \Omega$. There is a double cone $\mathcal{O}_{1}$ such that $\left[Q^{*}, Q(x)\right]=0$ for all $x \in \mathcal{O}_{1}^{\prime}$ (the spacelike complement of $\left.\mathcal{O}_{1}\right)$. Using the Jost-Lehmann-Dyson representation of $\left(\Omega, Q^{*} Q(x) \Omega\right)-\left(\Omega, Q(x) Q^{*} \Omega\right)$ and the properties of the solutions of the fivedimensional wave equation [11] one can show that

$$
\left(\Omega, Q^{*} P_{M} Q(x) \Omega\right)-\left(\Omega, Q(x) P_{M} Q^{*} \Omega\right)=0 \forall x \in \mathcal{O}_{1}^{\prime} .
$$

\footnotetext{
1 We shall omit the indices of the norms when no confusion is possible.
} 
Now one can apply the method of [3, Eqs. (3)-(15)] to estimate

$$
\left|\left(P_{M} \Psi, U(\boldsymbol{x}) P_{M} \Psi\right)\right| \leqq c^{2} e^{2 \kappa r} \varphi(|\boldsymbol{x}|) \forall M,
$$

where $\varphi(|\boldsymbol{x}|)$ decreases faster than any inverse power of $|\boldsymbol{x}|$. So we get

$$
\left|\boldsymbol{\nabla}_{\boldsymbol{p}}\left\|\left(P_{M} \Psi\right)(\boldsymbol{p})\right\|^{2}\right| \leqq \int d^{3} \boldsymbol{x}|\boldsymbol{x}| c^{2} e^{2 \kappa r} \varphi(|\boldsymbol{x}|)=f(c, r) .
$$

For a given $\varepsilon>0$, define $\delta=\varepsilon^{2} / f(c, r)$ and $P_{\delta}$ as the projection onto those states whose support in momentum space is contained in a $\delta$-neighbourhood of $q$. The linear mapping $l_{\boldsymbol{q}}: \mathscr{H} \supset P_{\delta} P_{\boldsymbol{M}} \mathscr{M}_{\boldsymbol{r}} \rightarrow h_{\boldsymbol{q}}: l_{\boldsymbol{q}}\left(P_{\delta} P_{M} \Psi\right)=\left(P_{\delta} P_{M} \Psi\right)(\boldsymbol{q})=\left(P_{\boldsymbol{M}} \Psi\right)(\boldsymbol{q})$ is uniformly bounded on those vectors with $\left\|\left(P_{M} \Psi\right)(q)\right\| \geqq \varepsilon$ : the estimate (3.6) gives $\left\|\left(P_{M} \Psi\right)(\boldsymbol{p})\right\|^{2} \geqq\left\|\left(P_{M} \Psi\right)(\boldsymbol{q})\right\|^{2}-f(c, r)|\boldsymbol{p}-\boldsymbol{q}|$,

$$
\begin{aligned}
\left\|P_{\delta} P_{M} \Psi\right\|^{2} & \geqq\left\|\left(P_{M} \Psi\right)(\boldsymbol{q})\right\|^{2}(4 \pi / 3) \delta^{3}-f(c, r) \pi \delta^{4} \\
& \geqq 1 / 3\left\|\left(P_{M} \Psi\right)(\boldsymbol{q})\right\|^{2} \pi \delta^{3}
\end{aligned}
$$

which shows that $l_{\boldsymbol{q}}$ is bounded.

The set $\left\{P_{\delta} P_{M} \Psi \mid \Psi \in \mathscr{M}_{r},\|\Psi\| \leqq c,\left\|\left(P_{M} \Psi\right)(\boldsymbol{q})\right\| \geqq \varepsilon\right\}^{-}$is compact as a closed subset of the compact set (by 2.8) $\left\{P_{\delta} P_{M} \Psi\right\}^{-}$. Since $l_{q}$ is continuous on this set, its image $\left\{\left(P_{M} \Psi\right)(\boldsymbol{q}) \mid \Psi \in \mathscr{M}_{r},\|\Psi\| \leqq c,\left\|\left(P_{M} \Psi\right)(\boldsymbol{q})\right\| \geqq \varepsilon\right\}^{-}$is compact for any $\varepsilon$; therefore $\left\{\left(P_{M} \Psi\right)(\boldsymbol{q}) \mid \Psi \in \mathscr{M}_{r},\|\Psi\| \leqq c\right\}^{-}$is compact for any $\boldsymbol{q}, r, c, M$.

\section{Singly Localized States}

We pointed out already in part IIa that in relativistic quantum theory even most of the well-localized states have no fixed localization region. Accordingly, the sets $\mathscr{E}_{r}$ of states which are "singly localized at time 0 with correlation radius $r$ " are not uniquely fixed. For the proof of the equivalence theorem, however, it is not necessary to know the sets $\mathscr{E}_{r}$ in detail. Therefore we will state only some of their important properties.

Let $\left\{K_{d}\right\}$ be any sequence of translation invariant operators measuring those spacelike correlations in the states which extend over a longer distance than $d$. An example is provided by the coincidence operators introduced in Chapter VI.

Since by $(2.5)$ the correlations for well-localized states decay very fast, one expects that for $d>r$ :

$$
\begin{aligned}
& \left\|K_{d} \Psi\right\| \leqq \chi(d-r)\|\Psi\| \forall \Psi \in \mathscr{M}_{r}, \\
& \chi(d)=A \exp (-\alpha d), \alpha>0 .
\end{aligned}
$$

If a state obeys the same inequalities (4.1) as the states in $\mathscr{M}_{r}$ do, it is surely singly localized with correlation radius $r$. This leads us to the following two properties that determine which states will at least belong to the sets $\mathscr{E}_{r}$. Let $V(\underset{\sim}{\boldsymbol{P}})$ be unitary functions of the momentum operator. They commute with all $K_{d}$.

E1: $V(\underset{\sim}{\boldsymbol{P}}) \mathscr{M}_{\boldsymbol{r}} \subset \mathscr{E}_{r} \forall V(\underset{\sim}{\boldsymbol{P}})$.

E2: For any $\varepsilon>0$ there is a radius $r^{\prime}$ such that for all $\Psi_{i} \in \mathscr{M}_{r},\left\|\Psi_{i}\right\| \leqq 1$, and for all $V_{i}(\underset{\sim}{\boldsymbol{P}})$

$$
V_{1}(\underset{\sim}{\boldsymbol{P}}) \Psi_{1}+V_{2}(\underset{\sim}{\boldsymbol{P}}) \Psi_{2} \in \mathscr{E}_{\boldsymbol{r}^{\prime}}+\mathscr{U}_{\varepsilon}
$$

$\mathscr{U}_{\varepsilon}$ is the ball of radius $\varepsilon$ in state space. 
Property E2 describes "restrained linearity" of the sets $\mathscr{E}_{r}$, which is analogous to property (2.3) for $\mathscr{M}_{r}$. E2 is surely true if $\left\|V_{1}(\boldsymbol{P}) \Psi_{1}+V_{2}(\underset{\sim}{\boldsymbol{P}}) \Psi_{2}\right\| \leqq \varepsilon$. In the opposite case,

$$
\begin{aligned}
\left\|K_{d}\left(V_{1}(\underset{\sim}{\boldsymbol{P}}) \Psi_{1}+V_{2}(\underset{\sim}{\boldsymbol{P}}) \Psi_{2}\right)\right\| & \leqq\left\|K_{d} \Psi_{1}\right\|+\left\|K_{d} \Psi_{2}\right\| \\
\leqq \chi(d-r)\left(\left\|\Psi_{1}\right\|+\left\|\Psi_{2}\right\|\right) & \leqq 2 / \varepsilon \chi(d-r)\left\|V_{1}(\underset{\sim}{\boldsymbol{P}}) \Psi_{1}+V_{2}(\underset{\sim}{\boldsymbol{P}}) \Psi_{2}\right\| \\
& \leqq \chi\left(d-r^{\prime}\right)\left\|V_{1}(\underset{\sim}{\boldsymbol{P}}) \Psi_{1}+V_{2}(\underset{\sim}{\boldsymbol{P}}) \Psi_{2}\right\| .
\end{aligned}
$$

For our purpose, the weak property E2 is sufficient. It is valid for a much wider class of functions $\chi$ than those of the form (4.2).

In non-relativistic quantum mechanics, the support of the states $f\left(\boldsymbol{x}_{1}, \ldots, \boldsymbol{x}_{n}\right) \in \mathscr{E}_{r}$ in the relative coordinates $\boldsymbol{x}_{i}-\boldsymbol{x}_{j}$ is contained in a ball of radius $2 r$. Separating off the total momentum $\boldsymbol{p}$ gives the wave function $\hat{f}\left(\boldsymbol{p} \mid \boldsymbol{x}_{1}-\boldsymbol{x}_{2}, \ldots, \boldsymbol{x}_{n-1}-\boldsymbol{x}_{n}\right) \in h_{\boldsymbol{p}}$. For all $\boldsymbol{p}$ there exists a wave function $g_{p} \in \mathscr{M}_{2 r}$, namely $g_{p}\left(\boldsymbol{x}_{1}, \ldots, \boldsymbol{x}_{n}\right)=$ $g_{\boldsymbol{p}}\left(\sum_{i=1}^{n} \boldsymbol{x}_{i}\right) \hat{f}\left(\boldsymbol{p} \mid \boldsymbol{x}_{1}-\boldsymbol{x}_{2}, \ldots, \boldsymbol{x}_{n-1}-\boldsymbol{x}_{n}\right)$, where the support of $g_{\boldsymbol{p}}(\boldsymbol{y})$ is contained in a ball of radius $2 r$. For sufficiently large $r$, one can choose $\tilde{g}_{\boldsymbol{p}}(\boldsymbol{p})=1$ and $\int d^{3} \boldsymbol{y}\left|g_{\boldsymbol{p}}(\boldsymbol{y})\right|^{2} \leqq 1$. Then

$$
\hat{g}_{p}\left(p \mid x_{1}-x_{2}, \ldots, x_{n-1}-x_{n}\right)=\hat{f}\left(p \mid x_{1}-x_{2}, \ldots, x_{n-1}-x_{n}\right)
$$

and $\left\|g_{\boldsymbol{p}}\left(\boldsymbol{x}_{1}, \ldots, \boldsymbol{x}_{n}\right)\right\|_{\mathscr{H}} \leqq\left\|\hat{f}\left(\boldsymbol{p} \mid \boldsymbol{x}_{1}-\boldsymbol{x}_{2}, \ldots, \boldsymbol{x}_{n-1}-\boldsymbol{x}_{n}\right)\right\|_{\hbar_{p}}$. Therefore it is plausible that in relativistic quantum theory one can assume the following much weaker property: Let $\Psi \in \mathscr{E}_{r}$. There exist a bound $c(r, \boldsymbol{p})$ and, for any $\boldsymbol{p}$, a vector $\Phi_{\boldsymbol{p}} \in \mathscr{M}_{2 r}$ such that $\left\|\Phi_{\boldsymbol{p}}\right\|_{\mathscr{H}} \leqq c(r, \boldsymbol{p})\|\Psi(\boldsymbol{p})\|_{\ell_{p}}$ and $\Phi_{\boldsymbol{p}}(\boldsymbol{p})=\Psi(\boldsymbol{p})$.

In theories which satisfy the compactness criterion, one can apply Lemma 3.1 to get property

E3: For all $M, r, c, p$ the sets

$$
\mathfrak{f}_{r}^{M}(\boldsymbol{p})=\left\{\left(P_{M} \Psi\right)(\boldsymbol{p}) \in h_{\boldsymbol{p}} \mid \Psi \in \mathscr{E}_{r},\|\Psi(\boldsymbol{p})\|_{\ell_{p}}<c\right\}^{-}
$$

are compact.

This property says what the maximum number of states contained in the sets $\mathscr{E}_{r}$ is.

The natural choice of the states, which are singly loaclized at time $t$ is $U(t) \mathscr{E}_{r}$.

\section{Equivalence of the Particle Definitions}

In this chapter we will prove our first main theorem:

Theorem 5.1. In a massive relativistic quantum theory which satisfies the compactness criterion (2.8) a state obeys the geometrical particle definition (Chapter I) if and only if its mass spectrum is discrete.

In non-relativistic quantum mechanics, Ruelle [10], and Amrein and Georgescu [8] proved an analogous theorem on the geometrical characterization of bounded systems. Parts of our proof parallel the one given in [8].

Proof. "if"-part: A state $\Psi$ with discrete mass spectrum can be approximated uniformely in time by a finite linear combination of eigenstates of the mass operator:

$$
\begin{aligned}
& \left\|\exp (i H t) \Psi-\sum_{l=1}^{N} V_{l}(\underset{\boldsymbol{P}}{\boldsymbol{P}}, t) \psi_{l}\right\|<\varepsilon / 3 \forall t, \\
& V_{l}(\underset{\sim}{\boldsymbol{P}}, t)=\exp \left\{i\left(\stackrel{\sim}{\sim}^{2}+m_{l}^{2} \mathbb{1}\right)^{1 / 2} t\right\}, M \Psi_{l}=m_{l} \Psi_{l} .
\end{aligned}
$$


For all $\Psi_{l}$ there are, by (2.4), a radius $r$ and vectors $\Phi_{l} \in \mathscr{M}_{r}$ such that $\left\|\Psi_{l}-\Phi_{l}\right\|<$ $\varepsilon / 3 N \forall l$. Property E2 (Chapter IV) ensures that we can find a radius $r^{\prime}$ and vectors $\Phi_{t}^{\prime} \in \mathscr{E}_{\boldsymbol{r}^{\prime}}$ such that

$$
\left\|\sum_{l=1}^{N} V_{l}(\underset{\sim}{\boldsymbol{P}}, t) \Phi_{l}-\Phi_{t}^{\prime}\right\|<\varepsilon / 3 \forall t .
$$

So $\left\|\exp (i H t) \Psi-\Phi_{t}^{\prime}\right\|<\varepsilon \forall t$, i.e. $\Psi$ satisfies the geometrical particle definition. "only if"-part: We will show that a state with purely continuous mass spectrum is orthogonal to any geometrical one-particle state. We need no special assumptions about the mass spectrum - it can contain a continuous singular part.

For fixed momentum $\boldsymbol{p}$, we define the set $g_{p} \subset h_{p}$ by $g_{p}=\left\{d \in h_{p} \mid\right.$ for any $\varepsilon>0 \exists r$, such that for all $t$ there is a $\Psi_{t} \in \mathscr{E}_{r}$ with $\left\|\Psi_{t}(\boldsymbol{p})\right\|=\|d\|, \| \exp (i H[\boldsymbol{p}] t) d-$ $\left.\Psi_{t}(\boldsymbol{p}) \|<\varepsilon\right\}$.

$\Psi(\boldsymbol{p}) \in g_{\boldsymbol{p}}$ for almost all $\boldsymbol{p}$ if $\Psi$ satisfies the geometrical particle definition.

As a consequence of the compactness criterion, for any $d \in g_{p}$ there is a finitedimensional projector $F \in \mathscr{B}\left(h_{p}\right)$ such that

$$
\|(\mathbb{1}-F) \exp (i H[\boldsymbol{p}] t) d\|<\varepsilon\|d\| \forall t .
$$

To prove this we choose $M$ such that $\left\|\left(\mathbb{1}-P_{M}\right) d\right\|<\|d\| \varepsilon / 4$ and $r$ so big that $\left\|\exp (i H[\boldsymbol{p}] t) d-\Psi_{t}(\boldsymbol{p})\right\|<\|d\| \varepsilon / 4$ for suitable $\Psi_{t} \in \mathscr{E}_{\boldsymbol{r}}, \quad\left\|\Psi_{t}(\boldsymbol{p})\right\|=\|d\|$. Then $\left\|\exp (i H[\boldsymbol{p}] t) d-\left(P_{M} \Psi_{t}\right)(\boldsymbol{p})\right\|<\|d\| \varepsilon / 2 \forall t$. According to property E3, the vectors $\left(P_{M} \Psi_{t}\right)(p)$ are all contained in the compact set $\mathfrak{f}_{r}^{M}(4.3)$, and by $(2.7)$ there is a finitedimensional projector $F \in \mathscr{B}\left(h_{p}\right)$ such that $\left\|(\mathbb{1}-F)\left(P_{M} \Psi_{t}\right)(p)\right\|<\|d\| \varepsilon / 2 \forall t$. This gives (5.1).

We denote by $h_{p}^{c}$ the subspace of $h_{p}$ corresponding to the continuous spectrum of the reduced mass operator $M[p]$ or the reduced Hamiltonian $H[p]$.

For a vector $\Phi$ with purely continuous mass spectrum, $\Phi(\boldsymbol{p})$ lies in $h_{\boldsymbol{p}}^{c}$ for almost all $\boldsymbol{p}$. Therefore it is sufficient to show that $h_{\boldsymbol{p}}^{c}$ is orthogonal to $g_{\boldsymbol{p}}$.

Lemma 5.2. Let $e \in h_{p}^{c}$ and $f \in h_{p}$ be given; then

$$
\lim _{T \rightarrow \infty}(2 T)^{-1} \int_{-T}^{T} d t|(f, \exp \{i H[\boldsymbol{p}] t\} e)|^{2}=0 .
$$

Proof. $H[p]=\int_{0}^{\infty} \mu d E(\mu)$ is the spectral decomposition of the reduced Hamiltonian on $h_{p}^{c}$.

$$
\begin{aligned}
& |(f, \exp (i H[\boldsymbol{p}] t) e)|^{2} \\
& =\iint \exp (i(\lambda-\mu) t) d(e, E(\lambda) f) d(f, E(\mu) e) \\
& =\iint_{\lambda, \mu \geqq 0,|\lambda-\mu| \leqq \delta}+\iint_{\lambda, \geqq 0,|\lambda-\mu| \geqq \delta} .
\end{aligned}
$$

The polar decomposition of the measure

$$
d(f, E(\mu) e)=d \varrho_{1}(\mu)-d \varrho_{2}(\mu)-i d \varrho_{3}(\mu)+i d \varrho_{4}(\mu)
$$

gives four positive bounded measures $\varrho_{j}(\mu), \int d \varrho_{j}(\mu) \leqq 1$. We estimate the first term in (5.2):

$$
\begin{aligned}
\left|\iint_{|\lambda-\mu| \leqq \delta}\right| & =\left|\int_{\mu \geqq 0} e^{i \mu t} d(f, E(\mu) e)(\{E(\mu+\delta)-E(\mu-\delta)\} e, \exp (i H[\boldsymbol{p}] t) f)\right| \\
& \leqq \sup _{\mu}\|\{E(\mu+\delta)-E(\mu-\delta)\} e\| \sum_{j=1}^{4} \int d \varrho_{j}(\mu) \forall t
\end{aligned}
$$


For $e \in h_{\boldsymbol{p}}^{c}, E(\lambda) e$ is uniformly strongly continuous in $\lambda$. For any $\varepsilon>0$, we can find a $\delta>0$ such that

$$
\left|\iint_{|\lambda-\mu| \leqq \delta}\right| \leqq 4 \sup _{\mu}\|\{E(\mu+\delta)-E(\mu-\delta)\} e\|<\varepsilon / 2 \forall t .
$$

The time-avarage of the second term in (5.2) is

$$
\begin{aligned}
& (2 T)^{-1} \int_{-T}^{T} d t \iint_{|\lambda-\mu| \geqq \delta} \exp \{i(\mu-\lambda) t\} d(e, E(\lambda) f) d(f, E(\mu) e) \\
& \leqq \iint_{|\lambda-\mu| \geqq \delta}|\sin \{(\mu-\lambda) T\} /((\mu-\lambda) T)| \sum_{j} d \varrho_{j}(\lambda) \sum_{l} d \varrho_{l}(\mu) \\
& \leqq 16 /(\delta \cdot T)<\varepsilon / 2 \text { for } T>32 /(\delta \varepsilon) .
\end{aligned}
$$

Corollary 5.3. Let $F \in \mathscr{B}\left(h_{p}\right)$ be a finite-dimensional projector. For all $e \in h_{p}^{c}$ $\lim _{T \rightarrow \infty}(2 T)^{-1} \int_{-T}^{T} d t\|F \exp (i H[\boldsymbol{p}] t) e\|^{2}=0$.

Remark. If $e$ has a Lebesgue-absolutely continuous mass spectrum, $\lim _{t \rightarrow \infty}\|F \exp (i H[\boldsymbol{p}] t) e\|^{2}=0$.

Lemma 5.4. If $d \in g_{p}$ and $e \in h_{p}^{c}$, they are orthogonal.

Proof.

$$
\begin{aligned}
|(d, e)|^{2} \\
=(2 T)^{-1} \int_{-T}^{T} d t \mid(d, \exp \{i H[\boldsymbol{p}] t\}(\mathbb{1}-F) \exp \{-i H[\boldsymbol{p}] t\} e) \\
\quad+\left.(d, \exp \{i H[\boldsymbol{p}] t\} F \exp \{-i H[\boldsymbol{p}] t\} e)\right|^{2} \\
\leq 2 \sup _{t}\|(\mathbb{1}-F) \exp \{i H[\boldsymbol{p}] t\} d\|^{2} \\
\quad+2(2 T)^{-1} \int_{-T}^{T} d t\|F \exp \{i H[\boldsymbol{p}] t\} e\|^{2} \forall T, F .
\end{aligned}
$$

For a given $\varepsilon>0$, we choose a finite-dimensional projector $F$ such that by (5.1)

$$
\|(\mathbb{1}-F) \exp \{i H[\boldsymbol{p}] t\} d\|^{2}<\varepsilon / 4 \forall t .
$$

For sufficiently large $T$, by Corollary 5.3,

$(2 T)^{-1} \int_{-T}^{T} d t\|F \exp (i H[\boldsymbol{p}] t) e\|^{2}<\varepsilon / 4$,

so that $|(d, e)|^{2}<\varepsilon$.

\section{Coincidence Arrangements of Counters}

In the following sections we will describe mathematically a coincidence measurement with counters by "coincidence operators". If the theory has a complete particle interpretation, we can show that the asymptotic number of localization centres is the particle number.

To simplify the notation, we deal with theories describing only one kind of particle with mass $m>0$. The energy-momentum spectrum is:

$$
\left\{p^{\mu}=0\right\} \cup\left\{p^{\mu} \mid p_{\mu} p^{\mu}=m^{2}, p^{0}>0\right\} \cup\left\{p^{\mu} \mid p_{\mu} p^{\mu} \geqq 4 m^{2}, p^{0}>0\right\} .
$$


We use the formulation of Haag-Ruelle (H-R) scattering theory [5] in terms of bounded, almost local operators [4].

A bounded operator $A$ is almost local if it can be approximated by local operators $A_{r} \in \mathscr{R}\left(\mathcal{O}_{r}\right)\left(\mathcal{O}_{r}\right.$ is the double cone around the origin, with a basis of radius $r$ ) such that

$$
\begin{aligned}
& \|A\|=\left\|A_{r}\right\|,\left\|A-A_{r}\right\| \leqq\|A\| \varphi(r), \\
& \varphi(r) \leqq 1, \lim _{r \rightarrow \infty} r^{N} \varphi(r)=0 \forall N .
\end{aligned}
$$

There exist bounded one-particle creation operators $A^{*}$ :

$A$ is almost local,

$$
\begin{aligned}
& A^{*} \Omega \in \mathscr{H}_{1}, A \Omega=0, \\
& (2 \pi)^{3 / 2}\left(2 \omega_{p}\right)^{-1 / 2}\left\langle\boldsymbol{p} \mid A^{*} \Omega\right\rangle=1 \forall \boldsymbol{p} \quad \text { with } \quad \omega_{p} \leqq E,\left\langle\boldsymbol{p} \mid A^{*} \Omega\right\rangle \in \mathscr{D}\left(\mathbb{R}^{3}\right),
\end{aligned}
$$

where $\omega_{p}=\left(\boldsymbol{p}^{2}+m^{2}\right)^{1 / 2}$ and $|\boldsymbol{p}\rangle$ is the improper one-particle vector with momentum $\boldsymbol{p}$. We use the normalization

$$
\langle\boldsymbol{p} \mid \boldsymbol{q}\rangle=2 \omega_{p} \delta^{3}(\boldsymbol{p}-\boldsymbol{q}) .
$$

The $l$-particle scattering states $\Psi_{l}^{\text {in }}, \Psi_{l}^{\text {out }}$ are limits of the H-R approximations

$$
\begin{aligned}
& \Psi_{l}(t)=\frac{1}{\sqrt{l !}} \int \prod_{i=1}^{l} d^{3} x_{i} f_{l}\left(t ; \boldsymbol{x}_{1}, \ldots, \boldsymbol{x}_{l}\right) A^{*}\left(\boldsymbol{x}_{1}, t\right) \ldots A^{*}\left(\boldsymbol{x}_{l}, t\right) \Omega, \\
& \Psi_{l}^{\text {in (out) }}=\underset{t \rightarrow-\infty(+\infty)}{s-\lim _{t}} \Psi_{l}(t),
\end{aligned}
$$

with wave functions

$$
f_{l}\left(t ; \boldsymbol{x}_{1}, \ldots, \boldsymbol{x}_{l}\right)=(2 \pi)^{-3 l / 2} \int \prod_{i=1}^{l}\left(d^{3} \boldsymbol{p}_{i} \exp \left(i \boldsymbol{p}_{i} \boldsymbol{x}_{i}-i \omega_{p_{l}} t\right)\right) \tilde{f}_{l}\left(\boldsymbol{p}_{1}, \ldots, \boldsymbol{p}_{l}\right) .
$$

If the $\tilde{f}_{l}\left(\boldsymbol{p}_{1}, \ldots, \boldsymbol{p}_{l}\right) \in \mathscr{D}\left(\mathbb{R}^{3 l}\right)$ have non-overlapping support in velocity space (i.e. the planes $\boldsymbol{p}^{i} / \omega_{p_{l}}=\boldsymbol{p}^{j} / \omega_{p_{j}}$ don't lie in the support of $\tilde{f}_{l}$ ) the sequence $\Psi_{l}(t)$ converges very fast:

$$
|t|^{N}\left\|\Psi_{l}(t)-\Psi_{l}^{\text {in (out })}\right\|<M_{N} \forall N, \forall t<0(t>0) .
$$

These non-overlapping scattering states form a dense set in $\mathscr{H}$ if the theory is asymptotically complete.

For the $l$-particle states with bounded energy in the free field theory, we will also use the representation (6.6). In that case $A^{*}$ is the free field, smeared with a suitable test function from $\mathscr{S}\left(\mathbb{R}^{4}\right)$, so that (6.3) and (6.4) are valid. [A Wightman field, smeared with a test function from $\mathscr{S}\left(\mathbb{R}^{4}\right)$, is an unbounded almost local operator.]

The decay properties of truncated vacuum expectation values (TVEVs) of almost local operators have been studied in the literature for the cases where all operators are bounded (e.g. [1]) or unbounded (e.g. [2]). We will need the following case:

Lemma 6.1. In a theory with mass gap $\kappa>0$, let $A_{l}$ be unbounded and $Q_{i}$ be bounded operators which are almost local. For all $N$, there are $M_{N}$ such that

$$
\left|\left(\Omega, \prod_{l=1}^{m_{1}} A_{l}\left(\boldsymbol{a}_{l}\right) \prod_{i=1}^{m_{2}} Q_{i}\left(\boldsymbol{a}_{i}\right) \prod_{j=1}^{m_{2}} A_{j}\left(\boldsymbol{a}_{j}\right) \Omega\right)_{T}\right| \leqq M_{N}(1+d(\underline{a}))^{-N}
$$

where $d(a)=\max \left\{\left|\boldsymbol{a}_{i}-\boldsymbol{a}_{j}\right|\right\}$ is the diameter of the set of points $\left\{\boldsymbol{a}_{i}\right\}$. 
To prove the statement, one approximates the unbounded almost local operators by unbounded local ones [i.e. Wightman fields tested with functions in $\left.\mathscr{D}\left(\mathbb{R}^{4}\right)\right]$. The polar decomposition of the latter, and the subsequent spectral resolution of its positive part yields a bounded local approximation if one cuts off the spectral integral. The cut-off parameter depends, for each $A_{l}$, on $d(a)$ and on the position of $A_{l}$ in the TVEV. Approximating the $Q_{i}$ by bounded local operators, one gets rapidly decreasing $T V E V$ s of local operators [1], thus suppressing the polynomially growing norms of the bounded local approximations of the $A_{l}$.

Now we describe the counters. They are localized observables with positive bounded expectation values which don't count the vacuum. Therefore we call any operator a "counter" which has the following properties:

$C$ almost local, $C \geqq 0, C \Omega=0,\|C\|=1$.

The product of two spacelike widely separated counter operators represents a coincidence circuit of the detectors. So we describe a coincidence arrangement of $n$ counters $C_{i}$ at time $t$ with minimal separation $d$ by "coincidence operators"

$$
K_{d}^{(n)}(t)=\int_{\left|z_{i}-z_{j}\right| \geqq d} d^{3} z_{1} \ldots d^{3} z_{n} C_{1}\left(z_{1}, t\right) \ldots C_{n}\left(z_{n}, t\right) .
$$

The space integration is chosen so that the expectation of $K_{d}^{(n)}(t)$ measures the probability that there are at least $n$ particles with minimal pairwise separation $d$ in the state.

The unbounded operators $K_{d}^{(n)}(t)$ are idealizations of measurements in bounded regions, so we determine the domain of $K_{d}^{(n)}(t=0)$ using the approximating sequence of bounded operators

$$
K_{d}^{(n)}\{R\}=\int_{\left|z_{i}-z_{j}\right| \geqq d,\left|z_{l}\right| \leqq R} d^{3} z_{1} \ldots d^{3} z_{n} C_{1}\left(z_{1}\right) \ldots C_{n}\left(z_{n}\right) .
$$

Let $\hat{\mathscr{D}}$ be $\hat{\mathscr{D}}=\left\{\psi \in \mathscr{H} \mid w\right.$ - $\lim _{R \rightarrow \infty} K_{d}^{(n)}\{R\} \Psi$ exists and

$$
\left.w-\lim _{R \rightarrow \infty} K_{d}^{(n)}\{R\}^{*} \Psi \text { exists }\right\} .
$$

$\hat{\mathscr{D}}$ is dense in $\mathscr{H}$, because it contains all vectors created from the vacuum by almost local operators. On $\hat{\mathscr{D}}$

$$
\hat{K}_{d}^{(n)}=w-\lim _{R \rightarrow \infty} K_{d}^{(n)}\{R\}
$$

is a closable operator whose closure defines $K_{d}^{(n)}(t=0)$ :

$$
K_{d}^{(n)}(t=0)=\hat{K}_{d}^{(n) * *} .
$$

The natural domain of $K_{d}^{(n)}(t)$ is

$$
\mathscr{D}\left(K_{d}^{(n)}(t)\right)=\exp (i H t) \mathscr{D}\left(K_{d}^{(n)}(0)\right) \text {. }
$$

One could define hermitian coincidence operators if one used the symmetrized product of counters in (6.11). They would differ by a bounded operator whose norm decreases faster than any inverse power of $d$.

Next we show that the states of bounded energy for the free massive field theory, and the non-overlapping scattering states with bounded energy in an $\mathrm{H}-\mathrm{R}$ scattering theory are contained in the domains of all coincidence operators. As the $K_{d}^{(n)}(t)$ are closed operators, we will construct a sequence $\Psi_{R} \in \mathscr{D}\left(K_{d}^{(n)}(t)\right)$ 
so that $s-\lim _{R \rightarrow \infty} \Psi_{R}=\Psi$ and $s-\lim _{R \rightarrow \infty} K_{d}^{(n)}(t) \Psi_{R}$ exists. Then $\Psi \in \mathscr{D}\left(K_{d}^{(n)}(t)\right)$ and $K_{d}^{(n)}(t) \Psi=$ $S-\lim _{R \rightarrow \infty} K_{d}^{(n)}(t) \Psi_{R}$

Lemma 6.2. Let $A_{1}^{*}, \ldots, A_{l}^{*}$ be almost local creation operators (i.e. $A_{i} \Omega=0$ ), $f\left(\boldsymbol{x}_{1}, \ldots, \boldsymbol{x}_{l}\right) \in L^{2}\left(\mathbb{R}^{3 l}, d^{3 l} x\right)$ and let $\Psi=\int d^{3} x_{1} \ldots d^{3} x_{l} f\left(x_{1}, \ldots, x_{l}\right) A_{1}^{*}\left(x_{1}\right) \ldots A_{l}^{*}\left(x_{l}\right) \Omega$. Then $\Psi \in \mathscr{D}\left(K_{d}^{(n)}(t)\right)$ and $\left\|K_{d}^{(n)}(t) \Psi\right\| \leqq M\|f\|_{2}$.

The lemma says that all states of bounded energy for the free field, and the $\mathrm{H}-\mathrm{R}$ approximations of scattering states at finite times with bounded energy are contained in the domain of any $K_{d}^{(n)}(t)$ [cf. (6.3)-(6.9)].

Proof. $\Psi_{R}=\int_{\left|x_{i}\right| \leqq R} \Pi d^{3} x_{j} f\left(x_{1}, \ldots, x_{l}\right) A_{1}^{*}\left(x_{1}\right) \ldots A_{l}^{*}\left(x_{l}\right) \Omega$ is a state which is created from the vacuum by an almost local operator. Therefore $\Psi_{R} \in \mathscr{D}\left(K_{d}^{(n)}(t)\right)$ for all $R, t$. To establish the strong convergence of $\lim _{R \rightarrow \infty} K_{d}^{(n)}(t) \Psi_{R}$, it is sufficient to show that

$$
\begin{aligned}
& \int \prod_{i} d^{3} \boldsymbol{x}_{i} \prod_{j} d^{3} \boldsymbol{x}_{j}^{\prime}\left|f\left(\ldots, \boldsymbol{x}_{i}, \ldots\right) \| \bar{f}\left(\ldots, \boldsymbol{x}_{j}^{\prime}, \ldots\right)\right| \\
& \cdot\left|\left(\Omega, \prod_{j} A_{j}\left(\boldsymbol{x}_{j}^{\prime}\right) K_{d}^{(n)}(t)^{*} K_{d}^{(\boldsymbol{n})}(t) \prod_{i} A_{i}^{*}\left(\boldsymbol{x}_{i}\right) \Omega\right)\right|<\infty .
\end{aligned}
$$

Because $\left|f\left(\ldots \boldsymbol{x}_{i} \ldots\right) \bar{f}\left(\ldots \boldsymbol{x}_{j}^{\prime} \ldots\right)\right| \leqq 1 / 2\left|f\left(\ldots \boldsymbol{x}_{i} \ldots\right)\right|^{2}+1 / 2\left|f\left(\ldots \boldsymbol{x}_{j}^{\prime} \ldots\right)\right|^{2}$ the expression is bounded by

$$
\begin{aligned}
& \int \prod_{i} d^{3} \boldsymbol{x}_{i}\left|f\left(\ldots \boldsymbol{x}_{i} \ldots\right)\right|^{2} \sup _{\boldsymbol{x}_{i}} \int \prod_{j} d^{3} \boldsymbol{x}_{j}^{\prime} \mid\left(\Omega, \Pi A\left(\boldsymbol{x}^{\prime}\right) K^{*} K \Pi A^{*}(\boldsymbol{x}) \Omega \mid\right. \\
& \leqq\|f\|_{2}^{2} \sup _{\boldsymbol{x}_{i}} \int \Pi d^{3} \boldsymbol{x}_{j}^{\prime} \Pi d^{3} z_{k} \Pi d^{3} z_{m}^{\prime} \\
& \quad \cdot\left|\left(\Omega, \Pi A_{j}\left(\boldsymbol{x}_{j}^{\prime}\right) \Pi C_{m^{\prime}}\left(z_{m}^{\prime}, t\right) \Pi C_{k}\left(z_{k}, t\right) \Pi A_{i}^{*}\left(\boldsymbol{x}_{i}\right) \Omega\right)\right| .
\end{aligned}
$$

In the decomposition of the vacuum expectation values ( $V E V \mathrm{~s})$ into truncated $V E V \mathrm{~s}$ (TVEVS) only those terms appear which contain at least one $A^{*}$ and one $A$, because $A$ and $C$ annihilate the vacuum. So one will never integrate over all variables which occur in one $T V E V$. The $T V E V$ s are continuous and rapidly decreasing functions of the relative variables, so the integrals and suprema exist.

The proof of Lemma 6.2 shows that the existence of the limit is independent of the number of counters. So $\Psi$ is in the domain of all $\left(K_{d}^{(n)}(t)^{*} K_{d}^{(n)}(t)\right)^{k}$. We need a better estimate:

Lemma 6.3. Let $\Psi$ be defined as in Lemma 6.2, but with bounded almost local creation operators $A_{i}^{*}$. Then

$$
\left\|\left(K_{d}^{(n)}(t)^{*} K_{d}^{(n)}(t)\right)^{k} e^{i H \tau} \Psi\right\|^{2} \leqq M\|f\|_{2}^{2}(1+|t-\tau|)^{3(4 n k+l)},
$$

$M$ is independent of $f, d, t$, and $\tau$.

Proof. As in the last proof, one has

$$
\begin{aligned}
& \left\|\left(K_{d}^{(n)}(t)^{*} K_{d}^{(n)}(t)\right)^{k} e^{i \boldsymbol{H} \tau} \Psi\right\|^{2} \\
& \leqq\|f\|_{2}^{2} \sup _{\boldsymbol{x}_{\imath}} \int \prod_{j=1}^{l} d^{3} \boldsymbol{x}_{j}^{\prime} \prod_{\lambda=1}^{4 n k} d^{3} z_{\lambda}\left|\left(\Omega, \Pi A_{j}\left(\boldsymbol{x}_{j}^{\prime}\right) \Pi C_{\lambda}\left(z_{\lambda}, t-\tau\right) \Pi A_{i}^{*}\left(\boldsymbol{x}_{i}\right) \Omega\right)\right|
\end{aligned}
$$

The integrand can be decomposed into $T V E V \mathrm{~s}$, which one estimates as usual [1], approximating the almost local operators by local ones which lie spacelike 
to each other. All TVEVs are majorized by a bounded function which decreases rapidly as soon as the relative coordinates are bigger than $|t-\tau|$. So any integration gives at most a factor $c(1+|t-\tau|)^{3}$.

Lemma 6.4. Let $\Phi^{\text {out }}$ be a non-overlapping scattering state of bounded energy. Then $\Phi^{\text {out }} \in \mathscr{D}\left(K_{d}^{(n)}(t)\right)$ for all $d, n, t$; and $\left\|K_{d}^{(n)}(t)\left(\Phi^{\text {out }}-\Phi(t)\right)\right\| t^{N}<M_{N}$ for all $N, t>0$ (and analogously for $\Phi^{\text {in }}$ ).

Proof. $\dot{\Phi}(\tau)$ is of the form $e^{i H \tau} \Psi$ as in Lemma 6.3. So $K_{d}^{(n)}(t) \dot{\Phi}(\tau)$ and $K_{d}^{(n)}(t)^{*} K_{d}^{(n)}(t) \dot{\Phi}(\tau)$ are strongly continuous in $\tau$, and for $\tau_{2} \geqq \tau_{1}$ one gets:

$$
\begin{aligned}
& \left\|K_{d}^{(n)}(t)\left(\Phi\left(\tau_{2}\right)-\Phi\left(\tau_{1}\right)\right)\right\|=\left\|\int_{\tau_{1}}^{\tau_{2}} d \tau K_{d}^{(n)}(t) \dot{\Phi}(\tau)\right\| \\
& \leqq \int_{\tau_{1}}^{\tau_{2}} d \tau\left\|K_{d}^{(n)}(t) \dot{\Phi}(\tau)\right\| \leqq \int_{\tau_{1}}^{\infty} d \tau\|\dot{\Phi}(\tau)\|^{1 / 2}\left\|K_{d}^{(n)}(t)^{*} K_{d}^{(n)}(t) \dot{\Phi}(\tau)\right\|^{1 / 2} .
\end{aligned}
$$

The first factor in the integrand decreases faster than any inverse power of $\tau$, the second is polynomially bounded in $\tau$ for fixed $t$, according to Lemma 6.3. The integral vanishes in the limit $\tau_{1} \rightarrow \infty$, and $s-\lim _{\tau \rightarrow \infty} K_{d}^{(n)}(t) \Phi(\tau)$ exists

$$
\left\|K_{d}^{(n)}(t)\left(\Phi^{\mathrm{out}}-\Phi(t)\right)\right\| \leqq \int_{t}^{\infty} d \tau\|\dot{\Phi}(\tau)\|^{1 / 2}\left\|K_{d}^{(n)}(t)^{*} K_{d}^{(n)}(t) \dot{\Phi}(\tau)\right\|^{1 / 2} .
$$

$|t-\tau| \leqq \tau$ if $\tau \geqq t \geqq 0$, so the integrand is, for all $t$, bounded by a function $h(\tau)$ which decreases faster than any inverse power of $\tau$. Thus $\int_{t}^{\infty} h(\tau) d \tau$ decreases faster than any inverse power of $t$.

\section{Sensitivity of Counters}

In the sequel, we will meet functions $\Gamma(p)$ which can be interpreted as the sensitivity of counters. First we will state some of their mathematical properties.

Let $C$ be a counter operator (6.10) and $|\boldsymbol{p}\rangle$ an improper one-particle vector of momentum $\boldsymbol{p}(6.5)$. Then

$$
\Gamma(\boldsymbol{p})=(2 \pi)^{3}\left(2 \omega_{p}\right)^{-1}\langle\boldsymbol{p}|C| \boldsymbol{p}\rangle
$$

is a well-defined differentiable function of $\boldsymbol{p}$ : The functional $\left(2 \omega_{p}\right)^{-1 / 2} C^{1 / 2}|\boldsymbol{p}\rangle$ is defined by

$$
\int d^{3} p\left(2 \omega_{p}\right)^{-1 / 2} C^{1 / 2}|\boldsymbol{p}\rangle \tilde{f}(\boldsymbol{p})=C^{1 / 2}|f\rangle .
$$

If the states $|f\rangle$ have bounded energy $E$, one can represent this functional by a vector-valued function of $\boldsymbol{p}$. To show this, let $A^{*}$ be an almost local one-particle creation operator (6.3) and (6.4); then

$$
\begin{aligned}
|f\rangle & =\int d^{3} \boldsymbol{x} f(\boldsymbol{x}) A^{*}(\boldsymbol{x}) \Omega, \\
C^{1 / 2}|f\rangle & =\int d^{3} \boldsymbol{x}(2 \pi)^{-3 / 2} \int d^{3} \boldsymbol{p} e^{i \boldsymbol{p} \boldsymbol{x}} \tilde{f}(\boldsymbol{p}) C^{1 / 2} A^{*}(\boldsymbol{x}) \Omega \\
& =\int d^{3} \boldsymbol{p} \tilde{f}(\boldsymbol{p}) \int d^{3} \boldsymbol{x}(2 \pi)^{-3 / 2} e^{i \boldsymbol{p x}} C^{1 / 2} A^{*}(\boldsymbol{x}) \Omega .
\end{aligned}
$$

The order of the $\boldsymbol{x}$ - and $\boldsymbol{p}$-integration may be interchanged because $\int_{\omega_{p} \leqq E} d^{3} \boldsymbol{p}|\tilde{f}(\boldsymbol{p})|<\infty$ and the $\boldsymbol{x}$-integrand is norm-integrable. The vector-valued function

$$
(2 \pi)^{-3 / 2} \int d^{3} \boldsymbol{x} e^{i \boldsymbol{p} x} C^{1 / 2} A^{*}(\boldsymbol{x}) \Omega=\left(2 \omega_{p}\right)^{-1 / 2} C^{1 / 2}|\boldsymbol{p}\rangle
$$


is infinitely often strongly differentiable because $\int d^{3} \boldsymbol{x}|\boldsymbol{x}|^{N}\left\|\left[C^{1 / 2}, A^{*}(\boldsymbol{x})\right]\right\|<\infty \forall N$. So $\Gamma(\boldsymbol{p}) \in C^{\infty}\left(\mathbb{R}^{3}\right)$, and

$$
\Gamma(\boldsymbol{p}) \leqq M_{E} \quad \text { on } \quad\left\{\boldsymbol{p} \mid \omega_{p} \leqq E\right\} .
$$

In the free theory, $\Gamma(\boldsymbol{p})$ is uniformly bounded because one can construct a sequence of operators

$A_{\boldsymbol{p}}^{*} \quad$ with $(2 \pi)^{3 / 2}\left(2 \omega_{\boldsymbol{p}}\right)^{-1 / 2}\left\langle\boldsymbol{p} \mid A_{\boldsymbol{p}}^{*} \Omega\right\rangle=1$ and $\int d^{3} \boldsymbol{x}\left\|C^{1 / 2} A_{\boldsymbol{p}}^{*}(\boldsymbol{x}) \Omega\right\|<M$ uniformly in $\boldsymbol{p}$.

One expects that for large times, $\langle f|C(\boldsymbol{x}, t)| f\rangle$ describes the probability of the particle to be at $\boldsymbol{x}$ multiplied by the sensitivity of the counter. Araki and Haag showed in $[4$, Theorem 4$]$ that

$$
\lim _{t \rightarrow \infty}\left\langle f\left|t^{3} C(\boldsymbol{v} t, t)\right| f\right\rangle=|\tilde{f}(\boldsymbol{p})|^{2} \Gamma(\boldsymbol{p}) \quad \text { where } \quad \boldsymbol{v}=\boldsymbol{p} / \omega_{p} .
$$

(The differentiability assumptions are always fulfilled.) Furthermore, it is easy to see that for all $t$

$$
\int d^{3} \boldsymbol{x}\langle f|C(\boldsymbol{x}, t)| f\rangle=\int d^{3} \boldsymbol{p}|\tilde{f}(\boldsymbol{p})|^{2} \Gamma(\boldsymbol{p}) .
$$

According to (7.4) and (7.5), $\Gamma(p)$ is the probability that the detector $C$ counts a particle whose momentum is concentrated around $\boldsymbol{p}$.

\section{Coincidence Measurements at Large Times}

In this section we investigate the expectation values of coincidence operators at large times. The results are collected in the following theorem, and their physical interpretation will be given in the next section.

Theorem 8.1. Let $\Psi$ be an outgoing non-overlapping scattering state of bounded energy. For all $K_{d}^{(n)}(t)$, there are operators $K_{d}^{(n)+}$ and $K^{(n)+}$ with $\left\|K_{d}^{(n)+} P_{E}\right\|<\infty$ and $\left\|K^{(n)+} P_{E}\right\|<\infty$ such that

$$
\begin{aligned}
& \lim _{t \rightarrow \infty}\left(\Psi, K_{d}^{(n)}(t) \Psi\right)=\left(\Psi, K_{d}^{(n)+} \Psi\right), \\
& \lim _{d \rightarrow \infty} d^{N}\left\|\left(K_{d}^{(n)+}-K^{(n)+}\right) P_{E}\right\|=0 \forall N .
\end{aligned}
$$

If all counters leave the one-particle space invariant,

$$
\left[C_{i}, P_{1}\right]=0
$$

one has

$$
s-\lim _{t \rightarrow \infty} K_{d}^{(n)}(t) \Psi=K_{d}^{(n)+} \Psi .
$$

$K^{(n)+}$ commutes with the outgoing particle number. If $\psi_{l}\left(\boldsymbol{p}_{1}, \ldots, \boldsymbol{p}_{l}\right)$ is the outgoing wave function of the l-particle component of the scattering state $\Psi$,

$$
\left(K^{(n)+} \Psi\right)_{l}\left(\boldsymbol{p}_{1}, \ldots, \boldsymbol{p}_{l}\right)=\left\{\begin{array}{l}
\frac{l !}{(l-n) !}\left[\prod_{i=1}^{n} \Gamma_{i}\left(\boldsymbol{p}_{i}\right) \psi_{l}\left(\boldsymbol{p}_{1}, \ldots, \boldsymbol{p}_{l}\right)\right] \\
0 \text { for } n>l .
\end{array} \text { symmetrized for } n \leqq l,\right.
$$


$\Gamma_{i}\left(\boldsymbol{p}_{i}\right)$ is the sensitivity of the counter $C_{i}[c f .(7.1)]$. The same results are valid for incoming scattering states with $t \rightarrow-\infty$ and for all states of bounded energy of the free massive field for $|t| \rightarrow \infty$.

Proof. The states $\Psi$ can be represented by almost local operators at time $t$, according to (6.6). For the free field, this is exactly true, and for a non-overlapping scattering state we know from Lemma 6.4 that the error is rapidly decreasing. Thus the wave functions $f_{l}\left(t ; \boldsymbol{x}_{1}, \ldots, \boldsymbol{x}_{l}\right)$ [cf. (6.8)] are the only time dependent quantities in the expectation values.

$$
\begin{aligned}
& \left(\Psi, K_{d}^{(n)}(t) \Psi\right)=\frac{1}{\sqrt{l ! l^{\prime} !}} \sum_{l, l^{\prime}}^{\mathrm{finit}} \int \prod_{i=1}^{l} d^{3} \boldsymbol{x}_{i} \prod_{j=1}^{l^{\prime}} d^{3} \boldsymbol{x}_{j}^{\prime} \int_{\left|z_{\lambda}-z_{\mu}\right| \geqq d} \prod_{k=1}^{n} d^{3} z_{k} \\
& \cdot f_{l}\left(t ; \boldsymbol{x}_{1}, \ldots, \boldsymbol{x}_{l}\right) \bar{f}_{l}^{\prime}\left(t ; \boldsymbol{x}_{1}^{\prime}, \ldots, \boldsymbol{x}_{l}^{\prime}\right)\left(\Omega, \prod_{j=1}^{l^{\prime}} A\left(\boldsymbol{x}_{j}^{\prime}\right) \prod_{k=1}^{n} C_{k}\left(z_{k}\right) \prod_{i=1}^{l} A^{*}\left(\boldsymbol{x}_{i}\right) \Omega\right) \\
& +O\left(t^{-\infty}\right) .
\end{aligned}
$$

The $V E V$ are decomposed into $T V E V$ s which decrease rapidly in the relative coordinates. Using Ruelle's estimates of the smooth solutions of the Klein-Gordon equation (e.g. [2, Chapter VI.4]) one immediately sees that asymptotically all terms in which more than one $A$ and one $A^{*}$ are contained within one $T V E V$ vanish.

$$
\begin{aligned}
& \lim _{t \rightarrow \infty}\left(\Psi, K_{d}^{(n)}(t) \Psi\right) \\
& =\lim _{t \rightarrow \infty} \sum_{l} \int \prod_{i=1}^{l}\left(d^{3} \boldsymbol{x}_{i} d^{3} \boldsymbol{x}_{i}^{\prime}\right) f_{l}\left(t ; \ldots \boldsymbol{x}_{i} \ldots\right) \bar{f}_{l}\left(t ; \ldots \boldsymbol{x}_{j}^{\prime} \ldots\right) \int_{\left|z_{\lambda}-z_{\mu}\right| \geqq d} \prod_{k=1}^{n} d^{3} z_{k} \\
& \quad \cdot \sum_{\sum m_{j}=n}\left(\Omega, A\left(\boldsymbol{x}_{1}^{\prime}\right) \prod_{\kappa=1}^{m_{1}} C_{\kappa}\left(z_{\kappa}\right) A^{*}\left(\boldsymbol{x}_{1}\right) \Omega\right) \ldots\left(\Omega, A\left(\boldsymbol{x}_{l}^{\prime}\right) \prod_{v=1}^{m_{l}} C_{v}\left(z_{v}\right) A^{*}\left(\boldsymbol{x}_{l}\right) \Omega\right) .
\end{aligned}
$$

The last sum extends over all possibilities of placing the $n$ counters at $l$ locations. The limit of (8.7) is obtained by changing the region of $z_{i}$-integration. In a given term, if the operators $C_{\lambda}\left(z_{\lambda}\right)$ and $C_{\mu}\left(z_{\mu}\right)$ are both in one $V E V$, the integration region of $z_{\lambda}-z_{\mu}$ remains unchanged: $\left|z_{\lambda}-z_{\mu}\right| \geqq d$; but if they are in different $V E V \mathrm{~s}$, there are no restrictions on the range of $z_{\lambda}-z_{\mu}$. We will show in a typical example that the error caused by this change vanishes asymptotically:

$$
\begin{aligned}
& \int \prod_{i=1}^{2}\left(d^{3} \boldsymbol{x}_{i} f_{i}\left(t ; \boldsymbol{x}_{i}\right) d^{3} \boldsymbol{x}_{i}^{\prime} \bar{f}_{i}^{\prime}\left(t ; \boldsymbol{x}_{i}^{\prime}\right)\right)\left\{\int_{\left|z_{1}-z_{k}\right| \geqq d}-\int_{\left|z_{1}-z_{2}\right| \geqq d}\right\} \prod_{j=1}^{3} d^{3} z_{j} \\
& \cdot\left(\Omega, A\left(\boldsymbol{x}_{1}^{\prime}\right) C_{1}\left(z_{1}\right) C_{2}\left(z_{2}\right) A^{*}\left(\boldsymbol{x}_{1}\right) \Omega\right)\left(\Omega, A\left(\boldsymbol{x}_{2}^{\prime}\right) C_{3}\left(z_{3}\right) A^{*}\left(\boldsymbol{x}_{2}\right) \Omega\right) .
\end{aligned}
$$

The region of $z$-integration is contained in the following set:

$$
G=\left\{\left(z_{1}, z_{2}, z_{3}\right)|| z_{1}-z_{3} \mid \leqq d\right\} \cup\left\{\left(z_{1}, z_{2}, z_{3}\right)|| z_{2}-z_{3} \mid \leqq d\right\} \subset \mathbb{R}^{9} .
$$

Substituting, in its first part, $z_{1}=z_{3}+z_{1}^{\prime}$, we can estimate (8.8) by

$$
\begin{aligned}
& \int d^{3} \boldsymbol{x}_{2}\left|f_{2}\left(t ; \boldsymbol{x}_{2}\right)\right| \sup _{\boldsymbol{x}_{1}}\left|f_{1}\left(t ; \boldsymbol{x}_{1}\right)\right| \prod_{i=1}^{2} \sup _{\boldsymbol{x}_{i}^{\prime}}\left|f_{i}^{\prime}\left(t ; \boldsymbol{x}_{i}^{\prime}\right)\right| \\
& \cdot \int d^{3} \boldsymbol{x}_{1} d^{3} \boldsymbol{x}_{1}^{\prime} d^{3} z_{2}\left|\left(\Omega, A\left(\boldsymbol{x}_{1}^{\prime}\right) C_{1}\left(z_{3}+z_{1}^{\prime}\right) C_{2}\left(z_{2}\right) A^{*}\left(\boldsymbol{x}_{1}\right) \Omega\right)\right| \\
& \cdot \int d^{3} \boldsymbol{x}_{2}^{\prime} d^{3} z_{3}\left|\left(\Omega, A\left(\boldsymbol{x}_{2}^{\prime}\right) C_{3}\left(z_{3}\right) A^{*}\left(\boldsymbol{x}_{2}\right) \Omega\right)\right| \int_{\left|z_{i}\right| \leqq d} d^{3} z_{1}^{\prime}
\end{aligned}
$$

(and analogously for the other part of $G$ ). Therefore (8.8) is bounded by $M(1+|t|)^{-3}$. 
The resulting expression is time-translation invariant, as is seen most easily in its momentum space representation:

$$
\begin{aligned}
& \lim _{t \rightarrow \infty}\left(\Psi, K_{d}^{(n)}(t) \Psi\right)=\left(\Psi, K_{d}^{(n)+} \Psi\right) \\
& =\sum l \int \prod_{i=1}^{l} d^{3} \boldsymbol{p}_{i}\left|\tilde{f}_{l}\left(\boldsymbol{p}_{1}, \ldots, \boldsymbol{p}_{l}\right)\right|^{2}(2 \pi)^{3 n} \\
& \cdot \sum \prod_{j} \int_{\left|z_{j_{j} \mid \geqq d}\right| \boldsymbol{z}_{j_{i}}-z_{j_{k} \mid \geqq d}} d^{3} z_{j_{2}} \ldots d^{3} z_{j_{m}}\left(2 \omega_{p_{j}}\right)^{-1}\left\langle\boldsymbol{p}_{j}\left|C_{j_{1}} C_{j_{2}}\left(z_{j_{2}}\right) \ldots C_{j_{m}}\left(z_{j_{m}}\right)\right| \boldsymbol{p}_{j}\right\rangle .
\end{aligned}
$$

To prove the norm convergence (8.2), we show first that the terms which are independent of $d$ are bounded. In these terms there is only one counter in a cluster (if $l \geqq n$ ). The norm of each of these terms is bounded by

$$
\prod_{i=1}^{n} \sup _{\boldsymbol{p}, \omega_{p} \leqq E}\left((2 \pi)^{3} /\left(2 \omega_{p}\right)\right)\left\langle\boldsymbol{p}\left|C_{i}\right| \boldsymbol{p}\right\rangle=\prod_{i=1}^{n} \sup _{\boldsymbol{p}, \omega_{p} \leqq E} \Gamma_{i}(\boldsymbol{p}) .
$$

By (7.3) this is finite.

If the matrix elements in the expansion (8.9) contain more than one counter, their contribution to $K_{d}^{(n)+}$ decreases in norm faster than any inverse power of $d$ : The norm is bounded by sums and products of expressions of the form

$$
\sup _{\boldsymbol{p}, \omega_{p} \leqq E}\left(2 \omega_{p}\right)^{-1}\left|\left\langle\boldsymbol{p}\left|C_{j_{1}} \int_{\substack{\left|z_{j_{i} \mid \geqq d}\right| z_{j_{i}}=z_{j_{k}} \mid \geqq d \\ d_{j_{2}} \ldots d^{3} z_{j_{m}}}} C_{j_{2}}\left(z_{j_{2}}\right) \ldots C_{j_{m}}\left(z_{j_{m}}\right)\right| \boldsymbol{p}\right\rangle\right| .
$$

Using the representation (7.2) for $C|\boldsymbol{p}\rangle$, the rapid decrease of (8.10) in $d$ follows from the decrease of $T V E V \mathrm{~s}$ of almost local operators. This completes the proof of (8.2) and (8.5).

We will only sketch the proof of (8.4). The methods used above yield, for nonoverlapping scattering states of bounded energy $\Phi, \Psi\left(\Phi, \Psi \in P_{E^{\mathscr{H}}} \mathscr{H}\right.$ in the freefield case)

$$
\lim _{t \rightarrow \infty}\left(\Phi, K_{d}^{(n)}(t) \Psi\right)=\left(\Phi, K_{d}^{(n)+} \Psi\right) \text { and }\left\|K_{d}^{(n)}(t) \Psi\right\|<M \quad \text { for } \quad t>0
$$

which gives $w-\lim _{t \rightarrow \infty} K_{d}^{(n)}(t) \Psi=K_{d}^{(n)+} \Psi$.

Using condition (8.3), one shows $\lim _{t \rightarrow \infty}\left\|K_{d}^{(n)}(t) \Psi\right\|=\left\|K_{d}^{(n)+} \Psi\right\|$ and therefore

$$
s-\lim _{t \rightarrow \infty} K_{d}^{(n)}(t) \Psi=K_{d}^{(n)+} \Psi
$$

\section{Particle Number as a Limit of Local Observables}

Now we will examine the physical content of Theorem 8.1. The operators $K_{d}^{(n)+}$ and $K^{(n)+}$ differ even for large $d$ because the counter operators are not exactly local, but overlap even if their separation is large. But this difference is physically irrelevant. Because of the norm convergence (8.2), for a given measurement accuracy $\varepsilon$, one can find a separation $d_{0}$ such that

$$
\left\|\left(K_{d}^{(n)+}-K^{(n)+}\right) P_{E}\right\|<\varepsilon \forall d \geqq d_{0} .
$$

The relation (8.1) shows that the results of coincidence measurements become asymptotically time-independent and independent of $d>d_{0}$ within a given precision $\varepsilon$. This means that the particles become arbitrarily widely separated from 
each other for sufficiently large times because the coincidence operators annihilate those parts of the states in which the particles have a separation less than $d$.

The increasing separation of the particles is due to their spreading and does not require non-overlapping support of their wave functions: even for two free particles with identical wave functions, $f\left(\boldsymbol{p}_{1}, \boldsymbol{p}_{2}\right)=g\left(\boldsymbol{p}_{1}\right) g\left(\boldsymbol{p}_{2}\right)$, the expectation of $K_{d}^{(n)}(t)$ indicates an arbitrarily large separation for sufficiently large times.

If the particle number $l$ is smaller than the number $n$ of counters, the coincidence measurements will give zero because a particle can hit at most one counter. If $l \geqq n$, the factor $l ! /(l-n)$ ! in (8.5) stems from the number of possibilities that $n$ counters are triggered by $l$ particles, whereas $\prod_{i} \Gamma_{i}\left(\boldsymbol{p}_{i}\right)$ takes account of the sensitivity of the counters. A coincidence arrangement measures asymptotically individual, widely separated particles.

One can use special counters $C$ with the property $\Gamma(\boldsymbol{p})=\gamma=$ const for $\omega_{p} \leqq E$ [an example is $C=\left\|A^{*} A\right\|^{-1} A^{*} A, A$ as in (6.3), (6.4)]. With these counters $K^{(n)+}$ becomes very simple ( $P_{l}$ is the projection on the $l$-particle space):

$$
K^{(n)+} P_{E}=\sum_{l \geqq n}^{\text {finite }}(l ! /(l-n) !) \gamma^{n} P_{l} P_{E}
$$

This gives for $P_{l}$ :

$$
P_{l} P_{E}=\left(\gamma^{l} l !\right)^{-1} \sum_{k=0}^{\text {finite }}(-1)^{k}(\gamma)^{-k}(k !)^{-1} K^{(l+k)+} P_{E} .
$$

For $n=1$ (one counter only)

$$
1 / \gamma K^{(1)+} P_{E}=\sum_{l=1}^{\mathrm{finite}} l P_{l} P_{E}
$$

is the particle number operator. The spectral resolution of $K^{(1)+}$ alone would also give $P_{l}$, but the representation (9.3) has the advantage that the physical interpretation is simpler. The coincidence operators annihilate those components in the state with less than $l$ particles, the coefficient in front of the sum gives the normalization 1 of the $l^{\text {th }}$ coincidence operator $(k=0)$ on the $l$-particle states, and the alternating coefficients in the sum ensure that the contributions of the coincidence operators cancel on states with particle number larger than $l$.

As a result of Theorem 8.1, one can obtain the asymptotic particle number of incoming or outgoing scattering states as a limit of local measurements at large times in large space regions. The particle number coincides with the asymptotic number of widely separated localization centres. As a special case, we recover our "geometrical particle definition": a one-particle state is always singly localized.

Acknowledgements. I am greatly indebted to Professor R. Haag for suggesting this problem and for his continuing encouragement and advice while the work was being done. Thanks are due to several members of the II. Institut für Theoretische Physik der Universität Hamburg for many helpful discussions, especially I thank Dr. D. Buchholz and Dr. M. Rinke.

\section{References}

1. Araki,H.: Einführung in die axiomatische Quantenfeldtheorie I. u. II. Unpublished Lecture Notes, Zürich: 1962

2. Jost, R.: The general theory of quantized fields. Providence, Rhode Island: Amer. Math. Soc. 1965

3. Haag, R., Swieca, J. A.: Commun. math. Phys. 1, 308-320 (1965)

4. Araki, H., Haag, R.: Commun. math. Phys. 4, 77-91 (1967) 
5. Haag, R.: Phys. Rev. 112, 669-673 (1958);

Ruelle, D.: Helv. Phys. Acta 35, 147-163 (1962);

Hepp, K.: Commun. math. Phys. 1, 95-111 (1965)

6. Schroer, B.: Fortschr. Phys. 11, 1-31 (1963)

7. Haag, R., Schroer, B.: J. Math. Phys. 3, 248-256 (1962);

Borchers, H.J.: N.Y.U. progress report, 1963

8. Amrein, W.O., Georgescu, V.: Helv. Phys. Acta 46, 635-658 (1973)

9. Streater, R.F., Wightman, A.S.: PCT, spin, and statistics and all that. New York: Benjamin 1964

10. Ruelle, D.: Nuovo Cimento 61A, 655-662 (1969)

11. See e.g. Hepp, K.: In: Chretien, M., Deser, S. (Eds.): Brandeis 1965, Vol. 1, p. 168ff. New York: Gordon and Breach 1966;

Doplicher,S., Haag, R., Roberts, J.E.: Lemma 6.2a in Commun. math. Phys. 35, 49 -85 (1974)

Communicated by R. Haag

Received January 6, 1975 ; in revised form June 10, 1975 